\title{
DISCONTINUOUS GROUPS ACTING ON
}

\section{HOMOGENEOUS SPACES OF REDUCTIVE TYPE}

\author{
TOSHIYUKI KOBAYASHI* \\ Department of Mathematics, University of Tokyo \\ Komaba, Meguro, Tokyo, 153, Japan \\ toshi\%tansei.cc.u-tokyo.ac.jp@relay.cs.net \\ and \\ Institute for Advanced Study, School of Mathematics \\ Princeton, NJ 08540, USA \\ toshi@math.ias.edu
}

Let $H$ be a closed subgroup of a Lie group $G$. The subject of this expository paper is roughly about the following:

Question A-0. How large a discrete subgroup of $G$ can act properly discontinuously on a homogeneous space $G / H$ ?

Our concern will be mainly with the case where $G / H$ is a homogeneous space of reductive type (Definition 5). If $H$ is not compact, the action of a discrete subgroup $\Gamma$ of $G$ on $G / H$ is not automatically properly discontinuous and the double coset $\Gamma \backslash G / H$ may be non-Hausdorff. This fact is the main difficulty in our problem. In fact, it may well happen that only finite subgroups of $G$ can act properly discontinuously on

*Partially supported by the Inamori foundation and DMS-9100383.

Typeset by $\mathcal{A}_{\mathcal{M}} \mathcal{S}-\mathrm{T}_{\mathrm{E}} \mathrm{X}$ 
$G / H$. For example, suppose that $G / H=S O(n+1,1) / S O(n, 1)$, a pseudo-Riemannian homogeneous space of metric type $(n, 1)$ and that $\Gamma$ is a discrete subgroup of $G$. Calabi and Markus proved that $\Gamma \backslash G / H$ is Hausdorff if and only if $\Gamma$ is a finite group [CalabiMarkus, 1962]. Thus, a homogeneous space $G / H=S O(n+1,1) / S O(n, 1)$ is somehow like a compact space. Named after their surprising discovery, such a homogeneous space is called to have a Calabi-Markus phenomenon.

In contrast to the above case with a noncompact isotropy subgroup $H$, Borel and Harish-Chandra [Borel-Harish-Chandra, 1962], [Borel, 1963] showed that Riemannian symmetric spaces are rich in properly discontinuous actions. That is, let $G$ be a real reductive linear Lie group and $K$ a maximal compact group of $G$. Then there exists a discrete subgroup $\Gamma$ of $G$ such that the double coset space $\Gamma \backslash G / K$ is a compact (Hausdorff smooth) manifold. Also, there exists a discrete subgroup $\Gamma$ such that the double coset space is noncompact manifold of finite volume.

On the other hand, even if the isotropy subgroup $H$ is noncompact, it may also happen that a homogeneous space has a large discontinuous group $\Gamma$ such that $\Gamma \backslash G / H$ is a compact manifold. Namely, this is an opposite extremal case to a Calabi-Markus phenomenon. A group manifold $G / H=G^{\prime} \times G^{\prime} / \operatorname{diag} G^{\prime}$ is the case. We want to find other homogeneous spaces which admit large discontinuous groups.

Now, let us fix some terminologies. Suppose that a discrete group $\Gamma$ acts continuously on a locally compact Hausdorff topological space $M$. For each subset $S$ of $M$, we put

$$
\Gamma_{S}=\{\gamma \in \Gamma: \gamma S \cap S \neq \emptyset\}
$$

Note that if $S$ is a singleton $\{p\}(p \in M)$ then $\Gamma_{S}$ is nothing but the isotropy subgroup at $p$. In general $\Gamma_{S}$ is merely a set.

Definition 1. The action of $\Gamma$ on $M$ is called properly discontinuous if $\Gamma_{S}$ is finite for 
any compact subset $S$ of $M$.

In the literature, there may be seen somewhat different definitions of proper discontinuity, and our definition here does not imply the freeness of the action (cf. [KulkarniRaymond, 1985] Footnote 5). Here the action of $\Gamma$ on $M$ is called free if and only if $\Gamma_{\{p\}}$ is trivial for any $p \in M$. In this paper, properly discontinuity is essentially important, but freeness is less important. In fact, if $\Gamma$ acts on a manifold $M$ properly discontinuously, then $\Gamma_{\{p\}}$ is not necessarily trivial indeed but is always finite. Correspondingly to it, $\Gamma \backslash M$ is not necessarily a smooth manifold but has a nice structure called $V$-manifold in the sense of [Satake, 1956]. Also if $\Gamma$ acts properly discontinuously on $M$, we can sometimes replace $\Gamma$ by a suitable subgroup $\Gamma^{\prime}$ of $\Gamma$ of finite index such that $\Gamma^{\prime}$ acts freely and properly discontinuously. The following result is useful to show the existence of such a subgroup.

Fact 1 ([Selberg, 1960] Lemma 8). A finitely generated matrix group has a torsionless subgroup of finite index.

Proper discontinuity implies by definition that every isotropy subgroup is finite and every $\Gamma$ orbit is closed. The latter condition corresponds to the fact that a single point is closed in the quotient topology of $\Gamma \backslash M$. In general, the converse implication does not hold.

Example 1. Suppose that $\Gamma:=\left\{\left(\begin{array}{ll}1 & n \\ 0 & 1\end{array}\right): n \in \mathbb{Z}\right\}, G:=S L(2, \mathbb{R})$ and $H:=$ $\left\{\left(\begin{array}{cc}a & 0 \\ 0 & a^{-1}\end{array}\right): a>0\right\}$. Let consider the action of $\Gamma$ on a hyperboloid $G / H$ of one sheet. Then every isotropy subgroup of $\Gamma$ is trivial and every $\Gamma$ orbit is closed in $G / H$. However, it can be shown that the $\Gamma$ action on $G / H$ is not properly discontinuous. 
Now let $H$ be a closed subgroup of a Lie group $G$ and consider the left action of $G$ (or its subgroup) on a homogeneous space $M=G / H$.

Definition 2. A discrete subgroup $\Gamma$ of $G$ is called a discontinuous group acting on $G / H$ if the action of $\Gamma$ on $G / H$ is properly discontinuous.

We should remark that $\Gamma$ is always assumed to be a subgroup of $G$ in this paper, so that any locally $G$-invariant structure is preserved when passing to $\Gamma \backslash G / H$. If we also treated an action of an abstract group, then the feature would become quite different. For instance, in the above Example 1, a hyperboloid $G / H$ is diffeomorphic to $S^{1} \times \mathbb{R}$ which clearly admits a free and properly discontinuous action of the integer group $\mathbb{Z}$. This action does not come from $G=S L(2, \mathbb{R})$. In fact, only finite subgroup can be a discontinuous group acting on $G / H$ (Calabi-Markus phenomenon) in this case.

Definition 3. Suppose that $\Gamma$ is a discontinuous group acting on $G / H$. We also assume that the $\Gamma$ action on $G / H$ is free, so that the double coset space $\Gamma \backslash G / H$ equips naturally with a manifold structure. If the double coset space $\Gamma \backslash G / H$ is compact, then $\Gamma$ is called a uniform lattice for $G / H$ and the quotient smooth manifold $\Gamma \backslash G / H$ is called a compact Clifford-Klein form of $G / H$. Suppose that $G / H$ carries a $G$-invariant measure. $\Gamma$ is called a lattice for $G / H$ if the double coset space $\Gamma \backslash G / H$ has a finite volume with respect to the measure induced from a $G$-invariant measure on $G / H$. $A$ non-uniform lattice for $G / H$ is a discrete subgroup of $G$ which is a lattice but is not a uniform lattice, so that $\Gamma \backslash G / H$ is a noncompact manifold of finite volume. These terminologies coincide with the familiar ones for group manifolds if we put $H=\{e\}$.

Taking two famous results due to Calabi and Markus, and Borel and Harish-Chandra into account, we can ask more explicitly as follows. 
Question A-1. When does there exist an infinite discontinuous group acting on $G / H$ $?$

If there exists such a discontinuous group, we want to ask furthermore,

Question A-2. When does there exist a compact Clifford-Klein form of $G / H$ ?

A simple remark here is that if $\Gamma$ is a uniform lattice for a group manifold $G$ and if $H$ is non-compact, then $\Gamma$ never acts properly discontinuously on $G / H$ and is not a uniform lattice for $G / H$. In this case the double coset space $\Gamma \backslash G / H$ is compact in the quotient topology indeed but this topology never becomes Hausdorff! Thus a uniform lattice for $G / H$ must be smaller than a uniform lattice for $G$ in some sense. In this respect, the cohomological dimension of an abstract group is a nice measure about the ‘size' of a discrete group ([Serre, 1971], see also [Kobayashi, 1989] Corollary 5.5).

The above Questions (A-1) and (A-2) are interesting from some viewpoints of different fields of mathematics:

First, from the view point of differential geometry, it is a fundamental problem that how local geometric structure restricts a global nature of a manifold. For instance, in Riemannian geometry, it has been an active area for long years to study how curvatures restrict fundamental groups of manifolds. In this connection our questions are restated as "what is a possible fundamental group of a manifold which is locally isomorphic to a particular homogeneous space ?" The result due to Calabi-Markus is a sort of such results in pseudo-Riemannian geometry, saying that the fundamental group of any relativistic spherical space form is finite.

Answers to these questions should involve some topological obstructions. For instance, let us consider pseudo-Riemannian structures. In contrast to Riemannian manifolds, not every smooth manifold carries a pseudo-Riemannian structure. For example, there 
does not exist a pseudo-Riemannian metric on the two dimensional sphere $S^{2}$ because a pseudo-Riemannian structure of metric type $(1,1)$ would generate a non-vanishing vector field. However, as is well known, there does not exist a non-vanishing vector field on $S^{2}$. This idea will be generalized later (see Theorem $5(1)$ ) by using the Euler class and a generalized Hirzebruch's proportionality principle.

Second, from the view point of non-commutative harmonic analysis or representation theory, Borel's result on the existence of compact Clifford-Klein forms (or noncompact ones of finite volume) of a Riemannian symmetric space has been a foundation on abundant theory such as Eisenstein series in harmonic analysis on square integrable functions over the double coset space. If there exist compact Clifford-Klein forms (or noncompact ones of finite volume) of a pseudo-Riemannian symmetric space, we could expect a theory of harmonic analysis on such a nice double coset manifold. I was benefited this motivation from the open problem posed by N.Wallach [Wallach, 1986].

In general the action of a discrete group is difficult to study. Instead the flow of some connected Lie subgroup sometimes helps us to understand the action of a discrete group. In this respect the following is a good (and easy) exercise:

Exercise 1. Find the topology of the quotient space $L \backslash G / H$ in Example 1 where $L:=\left\{\left(\begin{array}{ll}1 & x \\ 0 & 1\end{array}\right): x \in \mathbb{R}\right\} \subset G=S L(2, \mathbb{R})$.

The reader will find that this space $L \backslash G / H$ is 'locally' Hausdorff but not Hausdorff. Since $\Gamma \simeq \mathbb{Z}$ in Example 1 is a uniform lattice for a vector group $L \simeq \mathbb{R}$, the $L$ action reveals how proper discontinuity of the $\Gamma$ action fails (see Observation 1 below).

Here is a continuous analogue of proper discontinuity:

Definition 4. Suppose that a locally compact topological group $L$ acts continuously on a locally compact topological space $M$. For each subset $S$ of $M$, we put $L_{S}=$ 
$\{\gamma \in L: \gamma S \cap S \neq \emptyset\}$ as we defined for $\Gamma$. The the action of $L$ on $M$ is called proper if and only if $L_{S}$ is compact for every compact subset $S$ of $M$.

In the previous definition of proper discontinuity (Definition 2), compactness in Definition 4 was replaced by finiteness. The following elementary observation bridges between the action of a discrete group and that of a connected group.

Observation 1 ([Kobayashi, 1989], Lemma 2.3). Let a Lie group $L$ act on a locally compact space $M$ and $\Gamma$ be a cocompact discrete subgroup of $L$. Then

1) The $L$-action on $M$ is proper if and only if the $\Gamma$-action is properly discontinuous.

2) $L \backslash M$ is compact if and only if $\Gamma \backslash M$ is compact.

Suppose that $L$ and $H$ are closed subgroups of a Lie group $G$ and that the numbers of connected components of $L$ and $H$ are at most finite. Then our main interest in a continuous analogue is the following:

Question B-1. When is the action of $L$ on $G / H$ proper?

Question B-2. When is the double coset $L \backslash G / H$ compact in the quotient topology?

We notice that Question (A-2) on the existence of uniform lattices corresponds to the combination of Questions (B-1) and (B-2) in an continuous analogue.

In general the action of a noncompact Lie group is quite irregular in contrast to the one of a compact Lie group. R.S.Palais was the first person who emphasized the importance of the notion of properness in his study of the action of a non-compact Lie group [Palais, 1961]. The approach of a continuous analogue in a study of discontinuous groups acting on homogeneous spaces of reductive type was first taken by R.S.Kulkarni [Kulkarni, 1981], where he found that features of the groups which can act properly on $S O(p+1, q) / S O(p, q)$ dramatically depend on the conditions of $p$ and $q$ by making a 
detailed study of a quadratic form of type $(p+1, q)$. Our approach here is to pursue Kulkarni's idea through Questions (B-1) and (B-2). That is, we want to answer these questions in a continuous setting as generally as possible, and then we will apply these answers to discrete results. We shall carry out this program in the case of homogeneous spaces of reductive type (see Theorems 1 and 3 ).

In order to state explicit criteria of Questions (B-1) and (B-2), we fix some notations about a real reductive linear Lie group. Let $G$ be a connected real linear reductive Lie group, $\mathfrak{g}=\mathfrak{k}+\mathfrak{p}$ a Cartan decomposition of its Lie algebra. We fix a maximally abelian subspace $\mathfrak{a} \subset \mathfrak{p}$. $\mathfrak{a}$ is called a maximally split abelian subspace for $G$. If we want to emphasize the group $G$, we write $\mathfrak{a}_{G}$ for $\mathfrak{a}$. We denote by $W_{G}$ the Weyl group associated to the restricted root system of $\Sigma(\mathfrak{g}, \mathfrak{a}), c$-rank $G:=\operatorname{rank} K(\leq \operatorname{rank} G \geq$ ) $\mathbb{R}$-rank $G:=\operatorname{dim} \mathfrak{a} \leq \mathrm{d}(G):=\operatorname{dim} \mathfrak{p}$. Here $c$ - stands for compactness. Note that $\mathrm{d}(G)$ is the dimension of the associated Riemannian symmetric space $G / K$ of $G$. For example, if $G=S O_{0}(p, q)$, then $c$-rank $G=\left[\frac{p}{2}\right]+\left[\frac{q}{2}\right], \operatorname{rank} G=\left[\frac{p+q}{2}\right], \mathbb{R}-\operatorname{rank} G=$ $\min (p, q), \mathrm{d}(G)=p q$, and $W_{G} \simeq \mathfrak{S}_{l} \ltimes(\mathbb{Z} / 2 \mathbb{Z})^{l}$, where $l:=\mathbb{R}$-rank $G$ and $\mathfrak{S}_{l}$ denotes the $l$-th symmetric group.

Definition 5. Suppose that $H$ is a closed subgroup in $G$ with at most finitely many connected components. If there exists a Cartan involution of $G$ which stabilizes $H$, then $H$ is called reductive in $G$ and $G / H$ is called a homogeneous space of reductive type.

If $G / H$ is a homogeneous space of reductive type, then $G$ and $H$ have a realization in $G L(n, \mathbb{R})$ such that $H \subset G \subset G L(n, \mathbb{R})$ are closed subgroups and that $H={ }^{t} H$ and $G={ }^{t} G$. Here ${ }^{t} G:=\left\{{ }^{t} g: g \in G\right\}$. Also a chain rule holds: If $G_{0} \supset G_{1} \supset \cdots \supset G_{n}$ and if $G_{i-1} / G_{i}(1 \leq i \leq n)$ are all homogeneous spaces of reductive type, then so is $G_{0} / G_{n}$. The notion of a homogeneous space of reductive type contains important classes of homogeneous spaces such as semisimple symmetric spaces (or reductive symmetric 
spaces) and semisimple orbits in the Lie algebra under the adjoint action. We note that the 'compact form' of these homogeneous spaces are compact symmetric spaces, (generalized) flag varieties, respectively, which are apparently important and typical compact homogeneous spaces.

Remark 1. We avoid the terminology reductive homogeneous space which is usually used in the following sense: the Lie algebra $\mathfrak{g}$ may be decomposed into a vector space direct sum of the Lie algebra $\mathfrak{h}$ and a $H$-stable subspace $\mathfrak{m}$ (see for example, [KobayashiNomizu, 1969], Chapter X $\S 2$ ). This notion is wider than that of homogeneous spaces of reductive type in Definition 5. In particular neither $G$ nor $H$ itself is required to be reductive in this usual definition of reductive homogeneous space.

Taking this opportunity we would like to correct Definition-Lemma (2.6) in [Kobayashi, 1989]. There is no problem when $H$ is an algebraic subgroup defined over $\mathbb{R}$ (see [Mostow, 1955]). If $H$ is not algebraic but merely a closed Lie subgroup, then the condition (2.6.2) may be weaker than (2.6.1). The right definition there should be (2.6.1) that is the same with our definition here.

Suppose that $G / H$ is a homogeneous space of reductive type. Let $\mathfrak{a}_{H}$ be a maximally split abelian subspace for $H$. Then there exists an element $g$ of $G$ such that $\operatorname{Ad}(g) \mathfrak{a}_{H} \subset$ $\mathfrak{a}_{G}$. Put $\mathfrak{a}(H):=\operatorname{Ad}(g) \mathfrak{a}_{H}$, which is uniquely defined up to conjugacy of $W_{G}$. This notation will be convenient later, when we compare several subgroups at the same time.

Here is a criterion of the properness, which answers Question (B-1) ([Kobayashi, 1989] Theorem 4.1).

Theorem 1. Let $G / H, G / L$ be homogeneous spaces of reductive type. Then the following four conditions are equivalent:

1) $L$ acts on $G / H$ properly. 
1) $H$ acts on $G / L$ properly.

2) For any $g \in G, L \cap g H g^{-1}$ is compact.

$2)^{\prime} W_{G} \cdot \mathfrak{a}(L) \cap \mathfrak{a}(H)=\{0\}$.

In the above theorem, $(1) \Leftrightarrow(1)^{\prime},(2) \Leftrightarrow(2)^{\prime}$ and $(1) \Rightarrow(2)$ are trivial. The nontrivial part is the implication $(2)$ (or $\left.(2)^{\prime}\right) \Rightarrow(1)$. We shall observe this in Lemma 1 and Example 5 in a general setting.

Example 2. Let $G=S O(2 m, 2 n), L=U(m, n)$ and $H=S O(p, q) \simeq 1_{2 m-p} \times$ $S O(p, q) \times 1_{2 n-q} \subset G$. Here we suppose $0<q \leq p, 0<n \leq m, p \leq 2 m$ and $q \leq 2 n$. Then with a suitable coordinate, we can identify $\mathfrak{a}_{G}$ with $\mathbb{R}^{2 n}$ and $W_{G} \simeq \mathfrak{S}_{2 n} \ltimes(\mathbb{Z} / 2 \mathbb{Z})^{2 n}$ in $G L\left(\mathfrak{a}_{G}\right)$. Up to the conjugacy of an element of $W_{G}$, we have

$$
\begin{aligned}
\mathfrak{a}(L) & =\left\{\left(a_{1}, a_{1}, a_{2}, a_{2}, \ldots, a_{n}, a_{n}\right): a_{i} \in \mathbb{R}(1 \leq i \leq n)\right\} \\
\mathfrak{a}(H) & =\left\{\left(b_{1}, b_{2}, \ldots, b_{q}, 0, \ldots, 0\right): b_{i} \in \mathbb{R}(1 \leq i \leq q)\right\}
\end{aligned}
$$

So the condition $(2)^{\prime}$ in Theorem 1 amounts to $n=q=1$. Therefore we conclude that $L$ acts on $G / H$ properly if and only if $n=q=1$.

Thanks to the criterion $(2)^{\prime}$ of Theorem 1 , given a homogeneous space $G / H$ of reductive type, we can find a subgroup $L$ of $G$ that is isomorphic to $\mathbb{R}^{\mathbb{R} \text {-rank } G-\mathbb{R} \text {-rank } H}$ and that acts properly on $G / H$. This gives a complete answer to Question (A-1):

Corollary 1 (characterization of Calabi-Markus phenomenon). Let $G / H$ be a homogeneous space of reductive type. The followings are equivalent:

1) Any discontinuous group acting on $G / H$ is finite.

2) $\mathbb{R}-\operatorname{rank} G=\mathbb{R}-\operatorname{rank} H$. 
Example 3. There does not exists an infinite discontinuous group acting on semisimple symmetric spaces $G / H=G L(n, \mathbb{C}) / G L(n, \mathbb{R}), G L(n+m, \mathbb{R}) / G L(n, \mathbb{R}) \times G L(m, \mathbb{R})$, $U(m, n) / O(m, n)$, and etc. On the other hand, there exists a discontinuous group which is isomorphic to $\mathbb{Z}^{n}$ acting on semisimple symmetric spaces $S p(2 n, \mathbb{R}) / U(n, n)$, $G / H=G L(2 n, \mathbb{R}) / G L(n, \mathbb{C}), O(2 m, 2 n) / U(m, n)(n \leq m)$, and etc.

Historical Remarks 2. For the implication $(1) \Rightarrow(2)$, E. Calabi and L.Markus first proved in the case $G / H=S O(n+1,1) / S O(n, 1)$ in [Calabi-Markus, 1962]. Then J.A.Wolf extended their result to the case $G / H=S O(p+1, q) / S O(p, q)(q \leq p)$ in [Wolf, 1962]. After finding some other similar results [Wolf, 1964], he finally obtained the sufficiency of the real rank condition in the case of semisimple symmetric spaces in the 60's [Wolf, 1984]. His idea is also applicable to our more general setting. On the other hand, the proof of the necessity of the real rank condition given there is incomplete because of some confusion with the definition of proper discontinuity.

The converse implication $(2) \Rightarrow(1)$ is more difficult because we have to show the existence of an infinite discontinuous group acting on $G / H$ if $\mathbb{R}$-rank $H<\mathbb{R}$-rank $G$. It took about twenty years that the converse implication was first proved in the case $G / H=S O(p+1, q) / S O(p, q)(q>p)([$ Kulkarni, 1981] Theorem 5.7). The general case is due to [Kobayashi, 1989], Corollary 4.4 as an application of Theorem 1 with $\operatorname{dim} L=1$.

Corollary 1 in a reductive case should be in sharp contrast with a solvable group case:

Theorem 2 [Kobayashi, to appear (b)]. Let $G$ be a solvable group and $H$ a proper closed subgroup of $G$. Then there exists a discrete subgroup $\Gamma$ of $G$ that acts on $G / H$ properly discontinuously and freely and that the fundamental group $\pi_{1}(\Gamma \backslash G / H)$ is infinite. 
Loosely speaking, Calabi-Markus phenomenon does not occur in a large class of homogeneous spaces of solvable groups. Now we have made progress in two typical cases about Question (A-1), namely, reductive and solvable cases. So the problem for general case is to bridge the gap between these two typical cases. Here is an open problem.

Open problem 1. Let $G$ be a Lie group and $H$ be a proper connected subgroup of $G$. Then find a criterion deciding whether or not there exists an infinite discontinuous group acting on $G / H$ (see Definition 2).

A second application of Theorem 1 is to describe some structure of a discontinuous group acting on a semisimple group manifold of $\mathbb{R}$-rank 1 . Recall that, a discontinuous group acting on a group manifold $G$ means a discrete subgroup $\Gamma$ of $G \times G$ acting on $G \times G / \operatorname{diag} G$ properly discontinuously. First we make an observation:

Example 4. For a subgroup $\Phi$ of $G$ and for a homomorphism $\rho: \Phi \rightarrow G$, we put

$$
\Phi(\rho):=\{(\gamma, \rho(\gamma)): \gamma \in \Phi\}(\subset G \times G)
$$

If a homomorphism $\rho$ is a trivial representation $\mathbf{1}$, then the action of $\Phi(\mathbf{1})=\Phi \times 1$ on $G \simeq G \times G / \operatorname{diag} G$ is nothing but the one from the left. In this sense we might regard the action of $\Phi(\rho)$ as a 'perturbation' of the left action of $\Phi$. If $\Phi$ is a discrete subgroup of $G$ and and if the image $\rho(\Phi)$ is relatively compact, then $\Phi(\rho)$ is also a discontinuous group acting on a group manifold $G \times G / \operatorname{diag} G$.

Suppose that $\Phi \subset P S L(2, \mathbb{R})$ is a fundamental group of a compact Riemann surface $M$ of genus $g(\geq 2)$ and fix generators of the first homology group $H_{1}(M, \mathbb{Z}) \simeq \mathbb{Z}^{2 g}$. Then we find a moduli space of homomorphisms from $\Phi$ to $S O(2)$ as $\operatorname{Hom}(\Phi, S O(2)) \simeq$ $\mathbb{T}^{2 g}$. That is, $\lambda \in \mathbb{T}^{2 g}$ defines a homomorphism $\varphi_{\lambda}: \mathbb{Z}^{2 g} \rightarrow \mathbb{T} \simeq S O(2) \subset \operatorname{PSL}(2, \mathbb{R})$, 
and we get a homomorphism $\rho_{\lambda}: \Phi \rightarrow P S L(2, \mathbb{R})$ as a composition of $\varphi_{\lambda}$ and $\Phi \simeq$ $\pi_{1}(M) \rightarrow \Phi /[\Phi, \Phi] \simeq H_{1}(M, \mathbb{Z}) \simeq \mathbb{Z}^{2 g}$. Then $\Phi\left(\rho_{\lambda}\right)=\left\{\left(\gamma, \rho_{\lambda}(\gamma)\right): \gamma \in \Phi\right\}$ forms a family of uniform lattices (Definition 3) for a group manifold of $G \times G / \operatorname{diag} G$ parametrized by $\lambda \in \mathbb{T}^{2 g}$.

In the case of a reductive group $G$ of real rank one, a discontinuous group acting on $G$ is essentially of the following form:

Corollary 2, [Kobayashi, to appear (b)]. Let $G$ be a connected noncompact reductive linear Lie group. Then the following conditions are equivalent.

1) $\mathbb{R}-\operatorname{rank} G=1$

2) Any torsionless discontinuous group $\Gamma$ in a group manifold $G \times G / \operatorname{diag} G$ is of the following form up to switch of factor:

$$
\Gamma=\Phi(\rho) \equiv\{(\gamma, \rho(\gamma)): \gamma \in \Phi\}
$$

with a subgroup $\Phi \subset G$ and with a homomorphism $\rho: \Phi \rightarrow G$.

The implication $(1) \Rightarrow(2)$ in the above Corollary is a generalization of the result due to R.Kulkarni and F.Raymond for $G=S L(2, \mathbb{R})$ case in their study of 3 dimensional Lorentz manifolds and Seifert fiber spaces. (See Theorem 5.2 and Introduction in [Kulkarni-Raymond, 1985] for more about their geometric motivations.)

As we have observed, Theorem 1 has a wide application because of its simplicity of the criterion. Now let us analyse it for further understanding and aiming at an application in a more general homogeneous space.

Let $H, L$ be closed subgroups of a locally compact topological group $G$. If $L$ acts properly on $G / H$, then any $L$-orbit is closed with a compact isotropy group. In general, 
this condition is not sufficient for the properness of the $L$-action (cf. Example 1 and Exercise 1). However, we pick up the second condition because of its simplicity:

Definition 6. We call that the triplet $(L, G, H)$ has the property (CI) if and only if $L \cap g H g^{-1}$ is compact for any $g \in G$. We call that the triplet $(L, G, H)$ is proper if and only if $L$ acts properly on $G / H$.

Then it is easy to prove the following Lemma directly from the definition (see [Bourbaki, 1960] for the first part.)

Lemma 1. With notation as above, the following conditions are equivalent:

1) $(L, G, H)$ is proper,

$1)^{\prime}(H, G, L)$ is proper,

$1)^{\prime \prime}(\operatorname{diag} G, G \times G, H \times L)$ is proper,

which imply the following equivalent conditions.

2) (L, G,H) has the property (CI),

$2)^{\prime}(H, G, L)$ has the property (CI),

$2)^{\prime \prime}(\operatorname{diag} G, G \times G, H \times L)$ has the property (CI).

For actual calculation, it is much easier to check the property (CI) than properness. So we are interested in how and to what extent the property (CI) approximates properness.

\section{Example 5.}

1) Suppose that $G$ is a linear reductive Lie group, and that $H, L$ are closed subgroups reductive in $G$ (see Definition 5 ). From Theorem 1 , the property $(\mathrm{CI}) \Leftrightarrow$ properness. 
2) Suppose that $G$ is a linear reductive noncompact Lie group. Let $G=K A N$ be an Iwasawa decomposition and let $H:=A, L:=N$. Then the property (CI) is always satisfied for $(L, G, H)$, while $L$ never acts properly on $G / H$.

3) If $L$ is normal in $G$ and if $H L$ is closed, then the property (CI) $\Leftrightarrow$ properness.

4) Suppose that $G=G L(2, \mathbb{R}) \ltimes \mathbb{R}^{2}, H=G L(2, \mathbb{R})$. Then for any connected closed Lie subgroup $L$ of $G$, the property $(\mathrm{CI}) \Leftrightarrow$ properness.

If one is familiar to standard structural theory of semisimple Lie group, then it would be an illustrative exercise to give a proof of (2), which is quite easy. We also leave the proof of (3) to the reader which is straightforward from the definition.

So far, our interest has been mainly in reductive groups. On the other hand, in a non-reductive case Example 5 (4) which is a continuous analogue of a connected complete affine flat manifold (equivalently, a manifold represented as $\Gamma \backslash G / H$, where $H=G L(n, \mathbb{R})$ and $G=H \ltimes \mathbb{R}^{n}$ and $\Gamma$ is a discontinuous group acting on $G / H$ freely), there might be also a room for a generalization of the fact that the property $(\mathrm{CI}) \Leftrightarrow$ properness. We will give a proof of (4) in the Appendix, which is based on a classification of the maximal connected Lie groups $L$ of $G$ such that $(L, G, H)$ has the property (CI), any of which we shall find also proper.

It would lead us to a good understanding on proper actions to attack

Open Problem 2. Find a nice sufficient condition on the triplet $(L, G, H)$ that assures the property $(\mathrm{CI}) \Leftrightarrow$ properness.

A continuous analogue of a compact Clifford-Klein form is a compact double coset space $L \backslash G / H$ where the triplet $(L, G, H)$ is proper (Definition 6). Here is a criterion for the compactness which answers Question (B-2) ([Kobayashi, 1989] Theorem 4.7). 
Theorem 3. Under the equivalent conditions in Theorem 1, the followings are equivalent:

1) $L \backslash G / H$ is compact in the quotient topology.

2) $\mathrm{d}(L)+\mathrm{d}(H)=\mathrm{d}(G)$.

By virtue of Theorems 1 and 3 in a continuous setting, we obtain a sufficient condition for the existence of compact Clifford-Klein forms.

Theorem 4 ([Kobayashi, 1989] §4). Suppose that $G$ is a real reductive linear group and that $H$ and $L$ are both reductive in $G$. If the triplet $(G, L, H)$ satisfies both of the following conditions

$$
\begin{gathered}
\mathfrak{a}(L) \cap W_{G} \cdot \mathfrak{a}(H)=\{0\}, \\
\mathrm{d}(L)+\mathrm{d}(H)=\mathrm{d}(G),
\end{gathered}
$$

then $G / H$ admits compact Clifford-Klein forms. Also $G / H$ has noncompact ones of finite volume. So does $G / L$.

In fact, if both (4-a) and (4-b) are satisfied, then a torsionless cocompact discrete subgroup of $L$ (this always exists) is automatically a uniform lattice for $G / H$ (see Observation 1). Similarly, a torsionless co-volume finite discrete subgroup of $L$ is automatically a lattice for $G / H$.

We have a natural question, that is, the converse of Theorem 4:

Open problem 3. Suppose that $G / H$ is a homogeneous space of reductive type which admits a compact Clifford-Klein form. Is there a subgroup $L$ reductive in $G$ which satisfies the conditions both (4-a) and (4-b)?

As far as the author knows, Theorem 4 covers all known results on the existence of compact Clifford-Klein forms of homogeneous spaces of reductive type. For instance, if 
$H$ is compact, then we can take $L=G$. If $(G, H)=\left(G^{\prime} \times G^{\prime}, \operatorname{diag} G^{\prime}\right)$ so that $G / H$ is a group manifold, then we can take $L=G^{\prime} \times 1$ or $1 \times G^{\prime}$. These examples are stupid because Theorem 4 for these cases is nothing but a result in [Borel, 1963], which we use as a starting point in a proof of Theorem 4. The next examples are remarkable.

Corollary 3. The following homogeneous spaces admit both compact Clifford-Klein forms and non-compact Clifford-Klein forms:

1) a) $U(2,2 n) / S p(1, n), \quad$ b) $U(2,2 n) / U(1) \times U(1, n)$,

2) a) $S O(2,2 n) / U(1, n), \quad$ b) $S O(2,2 n) / S O(1,2 n)$,

3) a) $S O(4,4 n) / S p(1, n)$, b) $S O(4,4 n) / S O(3,4 n)$,

4) a) $S O(4,3) / G_{2(2)}, \quad$ b) $S O(4,3) / S O(2) \times S O(4,1)$.

Remark 3. Among these examples, (2-b), (3-b) (i.e. $S O(p+1, q) / S O(p, q)$ cases) were previously known by [Kulkarni, 1981] Theorem 6.1. From (1-a) to (3-b) see [Kobayashi, 1989] Proposition 4.9.

Conversely, we find two necessary conditions for the existence of a compact CliffordKlein form:

Theorem 5. Let $G / H$ be a homogeneous space of reductive type. If $G / H$ admits a compact Clifford-Klein form, then the following conditions must hold.

1) If $\operatorname{rank} G=\operatorname{rank} H$, then $c-\operatorname{rank} G=c-\operatorname{rank} H$.

2) There does not exist a closed subgroup $G^{\prime}$ reductive in $G$ satisfying the following two conditions:

$(5)(\mathrm{a})$

$(5)(\mathrm{b})$

$$
\begin{aligned}
& \mathfrak{a}\left(G^{\prime}\right) \subset W_{G} \cdot \mathfrak{a}(H), \\
& \mathrm{d}\left(G^{\prime}\right)>\mathrm{d}(H) .
\end{aligned}
$$


The first condition given in Theorem 5 is in [Kobayashi, 1989] Proposition 4.10 (see also [Kulkarni, 1981] Corollary 2.10 and [Kobayashi-Ono, 1990] Corollary 5 for partial results), which is derived from a comparison of the Euler characteristic between two compact homogeneous spaces associated to $G / H$, namely, a compact subspace $K / H \cap K$ and a compact real form $G_{U} / H_{U}$ by using a generalization of Hirzebruch's proportionality principle [Kobayashi-Ono, 1990].

The second one is given in [Kobayashi, to appear (a)], which is derived from a comparison among subgroups reductive in $G$ in terms of the cohomological dimension of a discrete subgroup. The condition looks somewhat complicated but turns out to be quite useful. We explain applications of Theorem 5 in the following examples, which also reveal the limitations of Theorems 4,5 and Corollary 3 about the existence of compact Clifford-Klein forms obtained so far.

Example 6. The simplest application of Theorem $5(2)$ is a comparison of $G / H$ with one point $G / G$ by taking $G^{\prime}:=G$, yielding the fact that if $\mathbb{R}$-rank $G=\mathbb{R}$-rank $H$ and if $G / H$ is not compact then $G / H$ does not have a compact Clifford-Klein form. Alternatively this fact follows directly from the criterion of Calabi-Markus phenomenon in Corollary 1.

Example 7. $G / H=S L(3, \mathbb{C}) / S L(2, \mathbb{C})$ does not admit a compact Clifford-Klein form from Theorem $5(2)$. In fact, we put $G^{\prime}:=S U(2,1)$ and then we have $W_{G} \cdot \mathfrak{a}\left(G^{\prime}\right)=$ $W_{G} \cdot \mathfrak{a}(H)$ and $\mathrm{d}\left(G^{\prime}\right)=4>\mathrm{d}(H)=3$. On the other hand, we cannot tell about the (non-)existence of a compact Clifford-Klein form of $G / H=S L(3, \mathbb{R}) / S L(2, \mathbb{R})$ from Theorem 5 .

Example 8. Suppose that $G / H=S O(i+j, k+l) / S O(i, k) \times S O(j, l)$, where $i \leq j, k, l$. From Theorem $5(2)$, if $G / H$ admits a compact Clifford-Klein form, then $G / H$ is 
compact, or $H$ is compact, or $0=i<l \leq j-k$. Moreover, Theorem 5 (1) requires $j k l \equiv 0 \bmod 2$.

Conversely, if $i=l=0$ or if $(i, j, k, l)=(0,2 n, 1,1),(0,4 n, 1,3),(0,4,2,1)$, then there exists a compact Clifford-Klein formof $G / H$ (see Corollary 3).

Example 9. Suppose that $G / H=S O^{*}(2 n) / U(l, n-l) . G / H$ does not admit a compact Clifford-Klein form if $3 l \leq 2 n \leq 6 l$ and if $n \geq 3$. It admits compact Clifford-Klein forms if $(n, l)=(4,1),(4,3),(2,1), l=0$ or $l=n$.

Example 10. Suppose that $G_{\mathbb{C}} / H_{\mathbb{C}}$ is a complex irreducible semisimple symmetric space. Then $G_{\mathbb{C}} / H_{\mathbb{C}}$ does not have compact Clifford-Klein forms unless $G_{\mathbb{C}} / H_{\mathbb{C}}$ is locally isomorphic to either a group manifold or one of $S O(2 n+2, \mathbb{C}) / S O(2 n+1, \mathbb{C})$ $S L(2 n, \mathbb{C}) / S p(n, \mathbb{C}),(n \geq 2)$ and $E_{6, \mathbb{C}} / F_{4, \mathbb{C}}$. We are left with the latter three cases about the existence of compact Clifford-Klein forms.

The following two Corollaries are systematic applications of Theorem 5 to a typical family of homogeneous spaces of reductive type, that is, a semisimple orbit and a semisimple symmetric space, respectively. See [Kobayashi, to appear (a)] for a proof and some table of homogeneous spaces without compact Clifford-Klein forms.

Corollary 4. Let $G$ be a real reductive linear Lie group, and $X$ a semisimple element of $\mathfrak{g}$. If $G \cdot X \simeq G / Z_{G}(X)$ admits a compact Clifford-Klein form, then the orbit $G \cdot X$ carries a $G$-invariant complex structure.

We can show that $G$ itself is not a complex Lie group under the assumption of Corollary 4. (Here we exclude the trivial case that $G / Z_{G}(X)$ is itself compact.) In fact $G / Z_{G}(X)$ can be realized as an elliptic orbit. In particular, via a $G$-invariant nondegenerate bilinear form on $\mathfrak{g}$ (which always exists), this orbit carries a $G$-invariant (pseudo-)Kähler structure which is compatible with the symplectic structure coming 
from the identification with the coadjoint orbit. It should be in sharp contrast that a hyperbolic orbit does not admit an infinite discontinuous group (Calabi-Markus phenomenon) by virtue of Corollary 1. From Corollary 3, $G / H=S U(2,2 n) / U(1, n)$, $S O(2,2 n) / U(1, n)$ and $S O(4,3) / S O(2) \times S O(4,1)$ are elliptic orbits in $\mathfrak{g}$ with compact complex quotient manifolds which are pseudo-Kähler via the Killing form.

Corollary 5. If a semisimple symmetric space $G / H$ admits a compact Clifford-Klein form, then the associated symmetric pair $\left(\mathfrak{g}, \mathfrak{h}^{a}\right)$ is basic in the $\epsilon$-family $F\left(\left(\mathfrak{g}, \mathfrak{h}^{a}\right)\right)$ in the sense of Oshima-Sekiguchi [Oshima-Sekiguchi, 1984].

A stupid remark is that the associated symmetric pair $\left(\mathfrak{g}, \mathfrak{h}^{a}\right)$ of a Riemannian symmetric pair $(\mathfrak{g}, \mathfrak{k})$ is $(\mathfrak{g}, \mathfrak{g})$ which is trivially basic, and that of a group manifold $\left(\mathfrak{g}^{\prime}+\mathfrak{g}^{\prime}, \operatorname{diag} \mathfrak{g}^{\prime}\right)$ is again a group manifold which is also basic. A non-trivial list can be seen in [Kobayashi, to appear (a)]. It is in sharp contrast that a para-Hermitian symmetric space does not admit an infinite discontinuous group (Calabi-Markus phenomenon) by virtue of Corollary 1. 


\section{Appendix. Homogeneous spaces of semi-direct product groups}

\section{A.1. semi-direct product}

We recall that a locally compact second countable group $G$ is called amenable if for every continuous $G$-action on a compact metrizable space $X$, there exists a $G$-invariant probability measure on $X$. Then we have:

Fact A.1.1 (see [Zimmer, 1984] Corollary 4.1.9). Suppose that $G$ is a connected Lie group. Then $G$ is amenable if and only if $G$ is a compact extension of a solvable group.

Proposition A.1.2. Let $G$ be a Lie group and $H$ be a closed subgroup. Assume that $\mathfrak{h}$ contains a maximal semisimple algebra of $\mathfrak{g}$. Then any connected closed subgroup $L$ such that $(L, G, H)$ has the property (CI) (Definition 6) is amenable.

Proof. Let $\mathfrak{l}=\mathfrak{l}_{s}+\mathfrak{l}_{n}$ be a Levi decomposition of $\mathfrak{l}$, where $\mathfrak{l}_{s}$ is a maximal semisimple algebra and $\mathfrak{l}_{n}$ is the radical. It follows from the assumption that there exists $g \in G$ such that $\mathfrak{l}_{s} \subset \operatorname{Ad}(g) \mathfrak{h}$. Thus, $L \cap g H g^{-1} \supset L_{s}$, where $L_{s}$ is a connected semisimple Lie subgroup with Lie algebra $\mathfrak{l}_{s}$. Therefore $L_{s}$ must be compact. $L$ is thus a compact extension of a solvable group, and therefore, an amenable group.

A typical example of $(G, H)$ with the assumption of Proposition (A.1.2) is the case where $H$ is reductive in $G L(n, \mathbb{R})$ (e.g. $H=G L(n, \mathbb{R}), S O(p, n-p)$ and etc) and $G=H \ltimes \mathbb{R}^{n}$. A conjecture due to L.Auslander is that a uniform lattice for $\mathbb{R}^{n} \simeq$ $G L(n, \mathbb{R}) \ltimes \mathbb{R}^{n} / G L(n, \mathbb{R})$ contains a solvable subgroup of finite index. G.A.Margulis (1983) showed that this conjecture is not true if one drops the compactness condition. Proposition (A.1.2) shows that a continuous analogue of this conjecture is quite easy to be proved in a more general setting, in particular without the compactness condition of $L \backslash G / H$. 


\section{A.2. affine transformation group of $\mathbb{R}^{2}$}

Let $G=G L(2, \mathbb{R}) \ltimes \mathbb{R}^{2}$, the affine transformation group of $\mathbb{R}^{2}$. The multiplicative structure is given by $\left(g_{1}, v_{1}\right) \cdot\left(g_{2}, v_{2}\right):=\left(g_{1} g_{2}, g_{1} v_{2}+v_{1}\right)$, where $g_{i} \in G L(2, \mathbb{R}), v_{i} \in \mathbb{R}^{2}$. as usual. The Lie algebra $\mathfrak{g}$ of $G$ is identified with

$$
M(3,2 ; \mathbb{R})=\left\{(A, u): A \in \mathfrak{g l}(2, \mathbb{R}), u \in \mathbb{R}^{2}\right\}
$$

equipped with $\left[\left(A_{1}, u_{1}\right),\left(A_{2}, u_{2}\right)\right]=\left(\left[A_{1}, A_{2}\right], A_{1} u_{2}-A_{2} u_{1}\right)$. The adjoint action is given by $\operatorname{Ad}((g, v))(A, u)=\left(g A g^{-1}, g u-g A g^{-1} v\right)$. Let $H=G L(2, \mathbb{R})$, the isotropy subgroup of $G$ at $0 \in \mathbb{R}^{2}$. Here is a classification of maximal connected Lie groups acting properly on $G / H \simeq \mathbb{R}^{2}$.

Proposition A.2.1. Up to conjugacy the maximal connected Lie subgroups of $G$ acting properly on $G / H$ are of the following forms;

$$
\begin{aligned}
& L_{1}=\left\{\left(\begin{array}{ccc}
e^{b} & 0 & a \\
0 & 1 & b
\end{array}\right): a, b \in \mathbb{R}\right\}, \\
& L_{2}=\left\{\left(\begin{array}{ccc}
1 & b & a \\
0 & 1 & b
\end{array}\right): a, b \in \mathbb{R}\right\}, \\
& L_{3}=\left\{\left(\begin{array}{ccc}
\cos \theta & -\sin \theta & a \\
\sin \theta & \cos \theta & b
\end{array}\right): a, b, \theta \in \mathbb{R}\right\} .
\end{aligned}
$$

It can be checked directly that $L_{i}$ acts properly on $G / H(i=1,2,3)$. Conversely, if a connected group $L$ acts properly on $G / H$, then $(L, G, H)$ has the property (CI). We shall classify $L$ such that $(L, G, H)$ has the property (CI) in the following way. First, $L$ is a compact extension of a solvable group from Proposition (A.1.2). In our case, a maximal compact subgroup of $G$ is of one dimension, and thus $L$ itself is a solvable Lie group. So we can take a sequence $0=\mathfrak{l}^{(0)} \triangleleft \mathfrak{l}^{(1)} \triangleleft \cdots \triangleleft \mathfrak{l}^{(n)}=\mathfrak{l}$ such that $\mathfrak{l}^{(i)}$ is a codimension one ideal in $\mathfrak{r}^{(i+1)}$. (It is easy to see that $n \leq 3$.) Now checking the 
property (CI) is reduced to the calculation of the normalizer $N_{\mathfrak{g}}\left(\mathfrak{l}^{(i)}\right)$ and to the case of $\operatorname{dim} L=1$ (Lemma (A.2.3)). The rest of this section is devoted to complete the proof of Proposition (A.2.1) by this procedure.

Lemma A.2.2. A complete representative of the adjoint orbit in $\mathfrak{g}$ is given by

$$
\begin{aligned}
X(a, b) & :=\left(\begin{array}{lll}
a & 0 & 0 \\
0 & b & 0
\end{array}\right) \quad(a, b \in \mathbb{R}, a \leq b), \quad W(a):=\left(\begin{array}{lll}
0 & 0 & 1 \\
0 & a & 0
\end{array}\right) \quad(a \in \mathbb{R}), \\
Y(a) & :=\left(\begin{array}{lll}
a & 1 & 0 \\
0 & a & 0
\end{array}\right) \quad(a \in \mathbb{R}), \\
Z(a, b) & :=\left(\begin{array}{lll}
a & -b & 0 \\
b & a & 0
\end{array}\right)(a, b \in \mathbb{R}, b>0) .
\end{aligned}
$$

Lemma A.2.3. Up to conjugacy, the one dimensional connected Lie subgroups of $G$ which act properly on $G / H$ have one of the following Lie algebras: $\mathbb{R} Z(0,1), \mathbb{R} W(1)$, $\mathbb{R} W(0), \mathbb{R} V$.

Proof. We notice that if $a \neq 0$ then there exists $g \in G$ such that $\operatorname{Ad}(g) \mathbb{R} W(a)=\mathbb{R} W(1)$. So the necessity is shown by checking the the property (CI). We have already seen the sufficiency before.

The proof of the following two lemmas is straightforward and so omitted.

Lemma A.2.4. The normalizers of the Lie algebras in Lemma (A.2.3) are given by,

$$
\begin{aligned}
& N_{\mathfrak{g}}(\mathbb{R} Z(0,1))=\mathbb{R} Z(0,1)+\mathbb{R} Z(1,0), \\
& N_{\mathfrak{g}}(\mathbb{R} W(0))=\left\{X \in M(3,2 ; \mathbb{R}): X_{21}=0\right\}, \\
& N_{\mathfrak{g}}(\mathbb{R} W(1))=\mathbb{R} W(1)+\mathbb{R} W(0), \\
& N_{\mathfrak{g}}(\mathbb{R} V) \quad=\mathbb{R} V+\mathbb{R} X(2,1)+\mathbb{R} W(0) .
\end{aligned}
$$


Set $W^{\prime}(a):=\left(\begin{array}{lll}a & 0 & 0 \\ 0 & 0 & 1\end{array}\right) \quad(a \in \mathbb{R}), V^{\prime}:=\left(\begin{array}{lll}0 & 0 & 1 \\ 0 & 0 & 0\end{array}\right)$, which are conjugate to $W(a), V$ respectively. Put $P:=N_{G}(\mathbb{R} W(0)), Q:=N_{G}(\mathbb{R} V) \subset G$.

\section{Lemma A.2.5.}

(1) $\operatorname{Ad}(G) Z(a, b) \cap \mathfrak{p}=\emptyset$ if $b \neq 0$.

(2) $\operatorname{Ad}(G) W(a) \cap \mathfrak{p}=\operatorname{Ad}(P) W(a) \coprod \operatorname{Ad}(P) W^{\prime}(a) \quad(a \in \mathbb{R})$.

(3) $\operatorname{Ad}(G) V \cap \mathfrak{p}=\operatorname{Ad}(P) V$.

(4) $\operatorname{Ad}(G) Z(a, b) \cap \mathfrak{q}=\emptyset$ if $b \neq 0$.

(5) $\operatorname{Ad}(G) W(a) \cap \mathfrak{q}= \begin{cases}\emptyset & \text { if } a \neq 0, \\ \mathbb{R}^{\times} W(0)=\operatorname{Ad}(Q) W(0) & \text { if } a=0 .\end{cases}$

(6) $\operatorname{Ad}(G) V \cap \mathfrak{q}=\coprod_{c \in \mathbb{R}} \operatorname{Ad}(Q)(V+c W(0))$.

Lemma A.2.6. Up to conjugacy the two dimensional connected Lie subgroups $L$ of $G$ which act properly on $G / H$ are of the following Lie algebras:

$\mathbb{R} W^{\prime}(0)+\mathbb{R} W(0), \mathbb{R} W^{\prime}(1)+\mathbb{R} W(0), \mathbb{R} V+\mathbb{R} W(0)$.

Proof. We have seen already that the corresponding Lie subgroups in Lemma (A.2.3) act properly on $G / H$. Let us verify the necessity part by the the property (CI). As $\mathfrak{l}$ is a solvable Lie algebra, we can assume that one of the Lie algebras in Lemma (A.2.3) is an ideal of $\mathfrak{l}$. First consider the case where $\mathbb{R} W(0) \triangleleft \mathfrak{l}$. Then $\mathfrak{l} \subset N_{\mathfrak{g}}(\mathbb{R} W(0))=\mathfrak{p}$. Up to conjugacy by $P$, we may assume that a complimentary subspace of $\mathbb{R} W(0)$ in $\mathfrak{l}$ is one of $\mathbb{R} W(0), \mathbb{R} W(1), \mathbb{R} W^{\prime}(0), \mathbb{R} W^{\prime}(1), \mathbb{R} V$ from Lemma (A.2.5). Then $\mathbb{R} W(0)$ is excluded because of linear dependency. $\mathbb{R} W(1)$ is also excluded because $\mathbb{R} W(0)+\mathbb{R} W(1)$ contains a subspace $\mathbb{R}(W(1)-W(0))$, whose corresponding connected Lie subgroup cannot act properly on $G / H$. The remaining is properly discontinuous cases. Similarly, we can treat the cases where $\mathbb{R} Z(0,1) \triangleleft \mathfrak{l}, \mathbb{R} W(1) \triangleleft \mathfrak{l}, \mathbb{R} V \triangleleft \mathfrak{l}$, yielding Lemma.

The final step is done similarly by using the following lemma. 
Lemma A.2.7. The normalizers of the Lie algebras in Lemma (A.2.6) are given by,

$$
\begin{aligned}
& N_{\mathfrak{g}}\left(\mathbb{R} W^{\prime}(0)+\mathbb{R} W(0)\right)=\mathfrak{g}, \\
& N_{\mathfrak{g}}\left(\mathbb{R} W^{\prime}(1)+\mathbb{R} W(0)\right)=\mathbb{R} X(1,0)+\mathbb{R} W^{\prime}(1)+\mathbb{R} W(0), \\
& N_{\mathfrak{g}}(\mathbb{R} V+\mathbb{R} W(0)) \quad=\mathbb{R} X(2,1)+\mathbb{R} Y(0)+\mathbb{R} V+\mathbb{R} W(0) .
\end{aligned}
$$

\section{REFERENCES}

[Borel, 1963]

A.Borel, Compact Clifford-Klein forms of symmetric spaces, Topology 2 (1963), 111-122.

[Borel-Harish-Chandra, 1962] A.Borel and Harish-Chandra, Arithmetic subgroups of algebraic groups, Ann. of Math. 75 (1962), 485-535.

[Bourbaki, 1960]

[Calabi-Markus, 1962]

[Kobayashi-Nomizu, 1969]

[Kobayashi-Ono, 1990]

[Kobayashi, 1989]

[Kobayashi, to appear (a)]

[Kobayashi, to appear (b)]

[Kulkarni, 1981]

[Kulkarni-Raymond, 1985]

[Mostow, 1955]

[Oshima-Sekiguchi, 1984]

[Palais, 1961]

[Satake, 1956]

[Selberg, 1960]
N.Bourbaki, Éléments de mathématique, Topologie générale, Chapitre 3, Hermann, Paris, 1960.

E.Calabi and L.Markus, Relativistic space forms, Ann. of Math. 75 (1962), 63-76.

S.Kobayashi and K.Nomizu, Foundations of differential geometry Vol.2, vol. 1,2, Interscience, John Wiley \& Sons, Inc., New York/London, 1969.

T.Kobayashi and K.Ono, Note on Hirzebruch's proportionality principle, J. Fac. Soc. Univ. of Tokyo 37-1 (1990), 71-87.

T.Kobayashi, Proper action on a homogeneous space of reductive type, Math. Ann. 285 (1989), 249-263.

A necessary condition for the existence of compact CliffordKlein forms of homogeneous spaces of reductive type, Duke Math. J. (to appear).

noncompact isotropy subgroups, (in preparation).

R.S.Kulkarni, Proper actions and pseudo-Riemannian space forms, Advances in Math. 40 (1981), 10-51.

R.S.Kulkarni and F.Raymond, 3-dimensional Lorentz space-forms and Seifert fiber spaces, J. Diff. Geometry 21 (1985), 231-268.

G.D.Mostow, Self-adjoint groups, Ann. of Math. 62 (1955), 44-55.

T.Oshima and J.Sekiguchi, The restricted root system of a semisimple symmetric pair, Advanced Studies in Pure Math. 4 (1984), 433-497.

R.S.Palais, On the existence of slices for actions of noncompact Lie groups, Ann. of Math. 73 (1961), 295-323.

I.Satake, On a generalization of the notion of manifold, Proc. Nat. Acad. Sci. U.S.A. 42 (1956), 359-363.

A.Selberg, On discontinuous groups in higher-dimensional symmetric spaces, Contributions to functional theory, Bombay, 1960, pp. 147-164. 
[Serre, 1971]

[Wallach, 1986]

[Wolf, 1962]

[Wolf, 1964]

[Wolf, 1984]

[Zimmer, 1984]
J.P.Serre, Cohomologie des groupes discrètes, Annals of Math. Studies, Vol.70, Princeton Univ. Press, Princeton, N.J., 1971, pp. 77-169.

N.R.Wallach, Two problems in the theory of automorphic forms, Open Problems in Representation Theory, pp. 39-40, Proceedings at Katata, 1986.

J.A.Wolf, The Clifford-Klein space forms of indefinite metric, Ann. of Math. 75 (1962), 77-80.

Isotropic manifolds of indefinite metric, Comment. Math. Helv. 39 (1964), 21-64. Inc., 1984

Spaces of constant curvature, 5-th edition, Publish of Perish,

R.J.Zimmer, Ergodic theory and semisimple groups, Birkhäuser, Boston, 1984. 